\title{
On the Robustness of Bayesian Fingerprinting Estimates of Global Sea Level Change ${ }^{\mathscr{O}}$
}

\author{
CARLING C. HAY \\ Department of Earth and Planetary Sciences, and Institute of Earth, Ocean, and Atmospheric Sciences, Rutgers, The \\ State University of New Jersey, Piscataway, New Jersey, and Department of Earth and Planetary Sciences, Harvard \\ University, Cambridge, Massachusetts \\ ERIC D. MORROW \\ Department of Earth and Planetary Sciences, and Institute of Earth, Ocean, and Atmospheric Sciences, Rutgers, The \\ State University of New Jersey, Piscataway, New Jersey \\ ROBERT E. KOPP \\ Department of Earth and Planetary Sciences, and Institute of Earth, Ocean, and Atmospheric Sciences, Rutgers, The \\ State University of New Jersey, Piscataway, and Rutgers Energy Institute, Rutgers, The State University of New \\ Jersey, New Brunswick, New Jersey \\ JERRY X. MITROVICA \\ Department of Earth and Planetary Sciences, Harvard University, Cambridge, Massachusetts
}

(Manuscript received 1 April 2016, in final form 12 January 2017)

\begin{abstract}
Global mean sea level (GMSL) over the twentieth century has been estimated using techniques that include regional averaging of sparse tide gauge observations, combining satellite altimetry observations with tide gauge records in empirical orthogonal function (EOF) analyses, and most recently the Bayesian approaches of Kalman smoothing (KS) and Gaussian process regression (GPR). Estimated trends in GMSL over 1901-90 obtained using the Bayesian techniques are $1.1-1.2 \mathrm{~mm} \mathrm{yr}^{-1}$, approximately $20 \%$ lower than previous estimates. It has been suggested that the adoption of a less restrictive subset of records biased the Bayesian-derived estimates. In this study, different subsets of records are used to demonstrate that GMSL estimates based on the Bayesian methodologies are robust to tide gauge selection. A method for determining the resolvability of individual sea level components estimated in a Bayesian framework is also presented and applied. It is found that the incomplete tide gauge observations result in posterior correlations between individual sea level contributions, making robust separation of the individual components impossible. However, various weighted sums of these components, as well as the total sum (i.e., GMSL), are resolvable. Finally, the KS and GPR methodologies allow for the simultaneous estimation of sea level at sites with and without observations. The first KS and GPR global maps of sea level change over the twentieth century are presented. These maps provide new estimates of twentieth-century sea level in data-sparse regions.
\end{abstract}

\section{Introduction}

Most recent estimates of global mean sea level (GMSL) rise over the first 90 years of the twentieth century are between 1.5 and $1.9 \mathrm{~mm} \mathrm{yr}^{-1}$ (e.g., Church and White

Supplemental information related to this paper is available at the Journals Online website: http://dx.doi.org/10.1175/JCLI-D-16-0271.s1.

Corresponding author e-mail: Carling C. Hay, carlinghay@ fas.harvard.edu
2011; Ray and Douglas 2011; Jevrejeva et al. 2008). These estimates are typically obtained using variants of one of two methodologies. The first approach regionally bins the temporally incomplete and spatially sparse tide gauge observations to obtain time series of regional means (e.g., Douglas 1997). Jevrejeva et al. (2008) extended this approach to better address the spatial sparsity in the tide gauge record. Their "virtual station" approach sequentially averages pairs of tide gauges, with a new virtual station located halfway between the original two stations. This method produces a more robust estimate of the 
uncertainty than when a single curve is adopted as being representative of regionwide sea level (Jevrejeva et al. 2008). The regional means from either approach are then averaged to compute a time series of GMSL. The second approach combines temporally long but spatially sparse tide gauge records with temporally short but nearly global altimetry observations of sea surface height (SSH) (Church and White 2006, 2011; Ray and Douglas 2011). Altimetry observations are used to obtain stationary empirical orthogonal functions (EOFs) that capture the dominant patterns of spatial variability observed in the approximate 20-yr altimetry record. The EOFs are then used, in conjunction with the tide gauges, to reconstruct the sea level field. GMSL estimates are computed as areaweighted averages of the global fields.

A limitation of GMSL estimates computed using regional binning is that biases may be introduced by the heterogeneous spatial distribution of tide gauges, which, over the twentieth century, were concentrated in the Northern Hemisphere (Holgate et al. 2013). The EOFbased approach attempts to mitigate this issue through the more complete spatial coverage of altimetry records. However, this approach assumes that the dominant spatial patterns observed in the satellite altimetry era (since about 1993) were statistically stationary over the entire twentieth century and that these short-term variability patterns represent the dominant features in long-term change. This assumption has been questioned on several grounds; for example, the covariance matrix used to constrain historical sea level is based on monthly satellite data, which may have different patterns of spatial variability than those present in long-term sea level records (Calafat et al. 2014). The method is also sensitive to the duration of the altimetry-based calibration period and prone to large biases (Christiansen et al. 2010). Moreover, the assumption of stationarity is at odds with observations indicating an evolving pattern, and variable rates, of melting land ice (e.g., Velicogna 2009; Leclercq et al. 2014) and increasing ocean heat content (Rhein et al. 2013) since the 1970s-80s. However, while using approximately $20 \mathrm{yr}$ of monthly altimetry data may affect EOF-based reconstructions of long-term sea level change, these reconstructions are more capable of resolving interannual variability.

A different approach to addressing data sparsity is to combine observations with process-based models of sea level geometries via a Bayesian framework. GMSL change over the twentieth century was dominated by two processes: thermal expansion of the oceans and melting of ice sheets and mountain glaciers (Cazenave and Llovel 2010; Milne et al. 2009). Melting ice sheets and glaciers give rise to distinct patterns of relative sea level change (i.e., changes in the SSH relative to the solid Earth surface; henceforth simply "sea level change").
These unique and nonuniform patterns (Clark and Lingle 1977; Clark and Primus 1987; Conrad and Hager 1997; Mitrovica et al. 2001; Tamisiea et al. 2001), or "fingerprints," can serve as the basis functions for linear models of sea level change due to changes in land ice volume (e.g., Mitrovica et al. 2001; Plag and Juttner 2001; Plag 2006). So-called static-equilibrium sea level changes (Farrell and Clark 1976; Dahlen 1976) arise from gravitational, deformational, and rotational perturbations to the elevation of the sea surface and sea floor associated with surface mass (ice plus water) redistribution; they do not include dynamic sea level changes arising from freshwater addition, which are less predictable and exhibit a shorter spatial scale of variability (Kopp et al. 2010). The first efforts to incorporate sea level fingerprints into estimates of GMSL change, and its individual sources, applied a stochastic inversion approach with assumed constant model coefficients, or rates of melt, over the twentieth century (Mitrovica et al. 2001). More recent approaches adopt a Bayesian methodology that naturally accommodates observation sparsity and nonstationarity in the estimated model parameters (Hay et al. 2013; 2015).

Using modified multimodel Kalman smoother (KS) and Gaussian process regression (GPR) approaches, Hay et al. (2015) fingerprinted twentieth-century tide gauge observations to estimate the underlying processes responsible for the geographic variability in sea level. After summing the individual contributions from thermal expansion and from mass changes of individual glaciers and ice sheets, they obtained revised GMSL rates of $1.2 \pm 0.2(\mathrm{KS})$ and $1.1 \pm 0.4 \mathrm{~mm} \mathrm{yr}^{-1}$ (GPR) over the time period 1901-90, approximately $20 \%-30 \%$ lower than previous estimates. These approaches extract global information from the sparse records and allow for the simultaneous estimation of sea level at sites with observations (i.e., tide gauge sites) and on a global grid of points where observations do not exist. In these methodologies, sea level changes due to local vertical land motion are modeled as spatially and temporally structured noise and are therefore not mapped into the GMSL estimates.

Understanding the $20 \%-30 \%$ difference between the Hay et al. (2015; hereafter H15) estimate and the results from earlier studies requires an examination of both the methodologies and the data employed. While all GMSL studies use tide gauge data archived at the Permanent Service for Mean Sea Level (PSMSL; Permanent Service for Mean Sea Level 2014; Holgate et al. 2013), the number of sites analyzed can differ significantly. The criteria for record selection often involve record length, continuity, and distance from areas experiencing vertical land movement due to tectonic activity, glacial isostatic adjustment (GIA), and other processes 
(e.g., Douglas 1997; Church et al. 2004; Holgate 2007). Additionally, analyses that use EOF reconstructions to estimate GMSL are restricted, by the orbit of the satellites, to only use tide gauges within about $\pm 65^{\circ}$ latitude (e.g., Church and White 2011; Ray and Douglas 2011). These different selection criteria result in vastly different subsets of tide gauges that can contain as few as 10 or as many as 600 sites. Hamlington and Thompson (2015) have suggested that tide gauge selection is largely responsible for the difference between the H15 GMSL estimate and previously published rates. However, the Hamlington and Thompson (2015) study did not implement either the KS or GPR methodologies. Here, we explore this issue more directly by using these methodologies to assess the sensitivity of the GMSL estimates to site selection.

A challenge faced by the H15 approaches, and indeed any fingerprint-based analysis (e.g., Mitrovica et al. 2001), is that, while the melt contributions to GMSL over the past century are on the order of $1 \mathrm{~mm} \mathrm{yr}^{-1}$, estimating the individual contributions to this total is a low signal-to-noise problem that is complicated by regional signals from steric effects (changes in sea surface height due to thermal expansion and salinity variations) and ocean dynamics (e.g., Cazenave and Llovel 2010; Kopp et al. 2010, 2015). The dynamic contribution to regional sea level changes may be of comparable, if not greater, magnitude to the melt contributions. The temporal incompleteness and geographic sparsity of tide gauges noted above also complicates efforts to estimate individual melt contributions. This incomplete sampling of a time-dependent, globally varying field limits the effective linear independence of the sea level fingerprint basis functions and thus the precision with which they can be uniquely separated. However, this limitation does not necessarily imply that a sum of the contributions that explicitly incorporates correlations among the individual contributions will lack robustness. As an example, Mitrovica et al. (2001) demonstrated that, while an Antarctic melt rate and a globally uniform signal could not be separated on the basis of the tide gauge records they analyzed, the sum of these two contributions was well constrained. We will investigate this issue within the context of the KS and GPR methodologies.

Section 2 summarizes the KS and GPR methods used to compute local sea level and GMSL. Section 3 explores the sensitivity of the KS GMSL estimate to tide gauge selection, focusing on three different subsets of tide gauges employed in previous analyses. In section 4, we use a Bayesian technique to discuss our ability to separate the individual melt contributions to GMSL. We also explore, using a simplified synthetic study, the extent to which total GMSL can be robustly estimated despite an inability to infer individual melt contributions. In section 5, we present and discuss the first global maps of the rate of sea level rise for the period 1901-90 estimated using the KS and GPR methodologies. Section 6 lists our conclusions.

\section{Methodologies}

The Bayesian methods employed by Hay et al. (2013, 2015), KS and GPR, compute posterior estimates of both GMSL and spatiotemporal sea level fields. They condition prior estimates that incorporate information about the spatiotemporal patterns of individual land-ice contributions and thermal expansion, as well as information from physical models of GIA and dynamic sea level, upon tide gauge observations. While the philosophy behind KS and GPR is similar, their implementation is not.

The Kalman smoother comprises three steps: the forward filtering pass, the backward smoother pass, and the multimodel component. In the forward filtering pass, the Kalman filter is run forward in time from 1880 to 2010. The Kalman filter consists of a series of equations that fall into two groups: time update equations and measurement update equations (Kalman 1960). The time update steps construct a prior estimate of sea level and ice sheet melt rates at time $k$, conditional upon the state of the system at time $k-1$. The state vector $\hat{\mathbf{x}}_{k}$ and its associated covariance $\mathbf{P}_{k}$ are projected forward in time using the state transition matrix $\boldsymbol{\phi}$ and the control input parameters $\mathbf{B} \mathbf{u}_{k}$ :

$$
\begin{aligned}
\hat{\mathbf{x}}_{k}^{-} & =\boldsymbol{\phi} \hat{\mathbf{x}}_{k-1}+\mathbf{B} \mathbf{u}_{k} \quad \text { and } \\
\mathbf{P}_{k}^{-} & =\boldsymbol{\phi} \mathbf{P}_{k-1} \boldsymbol{\phi}^{\mathrm{T}}+\mathbf{Q} .
\end{aligned}
$$

Here $\mathbf{Q}$ is the process noise covariance and the superscript minus sign indicates that the estimate is computed in the time update stage of the filter and represents the prior estimate of the states. The state vector in the H15 study comprises a vector of estimated sea levels at the 622 tide gauge sites and on a global $5^{\circ}$ grid and a vector of estimated melt rates for 22 ice sheets and mountain glaciers. The input control parameters include models of sea level changes due to GIA and ocean dynamics. The matrix $\boldsymbol{\phi}$ describes prior expectations about how sea level at the tide gauges and the ice sheet melt rates evolve between time steps $k$. Contained in $\phi$ are the spatial sea level fingerprints that connect local sea level to the equivalent eustatic rates that are being estimated (see methods in H15).

During the measurement update, the prior estimates of $\hat{\mathbf{x}}_{k}$ and $\mathbf{P}_{k}$ are conditioned upon the available observations $\mathbf{z}_{k}$ at time step $t_{k}$. The goal is to find a linear combination of the prior estimate $\hat{\mathbf{x}}_{k}^{-}$and a weighted 
difference between the observations and the prediction of the observations $\mathbf{H} \hat{\mathbf{x}}_{k}^{-}$, where $\mathbf{H}$ is a matrix that maps the state vector and covariance into the observation space:

$$
\begin{aligned}
\hat{\mathbf{x}}_{k} & =\hat{\mathbf{x}}_{k}^{-}+\mathbf{K}_{k}\left(\mathbf{z}_{k}-\mathbf{H} \hat{\mathbf{x}}_{k}^{-}\right) \text {and } \\
\mathbf{P}_{k} & =\left(\mathbf{I}-\mathbf{K}_{k} \mathbf{H}\right) \mathbf{P}_{k}^{-} .
\end{aligned}
$$

Here $\mathbf{I}$ is the identity matrix, and $\mathbf{K}_{k}$ is the Kalman gain matrix defined as follows:

$$
\mathbf{K}_{k}=\mathbf{P}_{k}^{-} \mathbf{H}^{\mathrm{T}}\left(\mathbf{H} \mathbf{P}_{k}^{-} \mathbf{H}^{\mathrm{T}}+\mathbf{R}\right)^{-1},
$$

where $\mathbf{R}$ is the observation noise covariance (Kalman 1960).

Once the forward pass is complete, the filter is then run backward in time from 2010 to 1880 and a linear combination of the two passes is calculated. This is the second step of the multimodel KS, and it ensures that the posterior estimates of the state vector and its covariance over the entire time window are conditioned upon all observations (Gelb et al. 1974).

The final step is the multimodel component. In this step, the forward and backward passes of the Kalman filter are run for multiple combinations of GIA and ocean dynamics models. The likelihood of each model combination given the tide gauge observations is computed, and a weighted sum of the results is obtained (Blom and Bar-Shalom 1988). This weighted sum results in the final KS estimate presented in H15.

In the Gaussian-process regression approach, the prior estimate of the spatiotemporal sea level field is simultaneously conditioned on all observations at all time points. The sea level field comprises contributions from ongoing GIA $\mathbf{f}_{\mathrm{GIA}}$, land-based ice mass $\operatorname{loss} \mathbf{f}_{M}$, and local effects $\mathbf{f}_{\mathrm{LSL}}$, including ocean dynamics, tectonics, and other nonclimate sources:

$$
\mathbf{f}(\mathbf{x}, t)=\mathbf{f}_{\mathrm{GIA}}(\mathbf{x}, t)+\mathbf{f}_{M}(\mathbf{x}, t)+\mathbf{f}_{\mathrm{LSL}}(\mathbf{x}, t),
$$

where $\mathbf{x}$ and $t$ specify the location and time, respectively. Each of the components has a Gaussian-process prior with the mean and covariance defined by the underlying physics of the respective process. For the GIA contribution, the prior mean and prior covariance are computed as the sample mean and sample covariance over 161 GIA model predictions. The zero-mean, spatiotemporally separable prior for the mass loss component has a spatial covariance computed from the sea level fingerprints associated with each melt source and a temporal covariance estimated from previous estimates of ice melt not based upon sea level observations. The prior for the local sea level component is the sum of a Gaussian process with a mean and covariance representing the sample distribution from six GCM outputs and a zero-mean Gaussian process with a Matérn covariance function that represents other nonclimatic factors (see methods in H15).

The Gaussian distribution of the total sea level field is partitioned into 1) individual space-time points that are observed $\mathbf{y}$ with some uncertainty characterized by a zero-mean white noise process with a covariance $\boldsymbol{\Sigma}_{p}$ and 2) those that are from the unobserved field $\mathbf{f}_{2}$ :

$$
\left[\begin{array}{c}
\mathbf{y} \\
\mathbf{f}_{2}
\end{array}\right] \sim \mathcal{N}\left(\left[\begin{array}{l}
\boldsymbol{\mu}_{1} \\
\boldsymbol{\mu}_{2}
\end{array}\right],\left[\begin{array}{cc}
\mathbf{K}_{11}+\mathbf{\Sigma}_{p} & \mathbf{K}_{12} \\
\mathbf{K}_{12}^{\mathrm{T}} & \mathbf{K}_{22}
\end{array}\right]\right) .
$$

From this joint distribution between the observations and the underlying field at specified coordinates, the posterior distribution of the underlying field can be computed from the posterior predictive distribution conditioned on the observations as follows:

$$
\mathbf{f}_{2}=\mathcal{N}\left(\boldsymbol{\mu}_{2}+\mathbf{K}_{12}^{\mathrm{T}}\left[\mathbf{K}_{11}+\boldsymbol{\Sigma}_{p}\right]^{-1} \mathbf{y}, \mathbf{K}_{22}-\mathbf{K}_{12}^{\mathrm{T}}\left[\mathbf{K}_{11}+\boldsymbol{\Sigma}_{p}\right]^{-1} \mathbf{K}_{12}\right)
$$

The outputs of the KS and GPR methodologies are reconstructions of the entire spatiotemporal sea level field, including the 622 tide gauge sites, and estimates of ice sheet melt rates over the analysis time window. Both techniques are able to produce estimates of sea level that match the tide gauge observations and provide statistically significant estimates of GMSL (H15). The robustness of the $\mathrm{H} 15$ techniques to tide gauge selection and observation sparsity derives from their bottom-up, process-based approach to estimating GMSL, which is significantly different from previous studies.

\section{Sensitivity tests}

\section{a. Alternative tide gauge subsets}

The GMSL results presented in $\mathrm{H} 15$ were obtained using 622 tide gauge records from the Revised Local Reference (RLR) database at the PSMSL (Fig. 1a). One of the goals of both the KS and GPR methodologies was to utilize as many tide gauges as possible; therefore, all sites within the RLR database that have at least $30 \mathrm{yr}$ of data between 1950 and 2010 were used, except for sites that are flagged as unreliable within the database. The minimum of $30 \mathrm{yr}$ of data criteria was imposed to ensure accurate computation of the observation covariance matrix, which is an input to both methodologies (see Hay et al. 2013; 2015).

Other studies have employed less inclusive criteria when selecting tide gauge sites. In particular, Church and White (2011; hereafter CW2011) and Ray and Douglas (2011; hereafter RD2011) were restricted to using tide gauge sites within the latitudinal range of the 

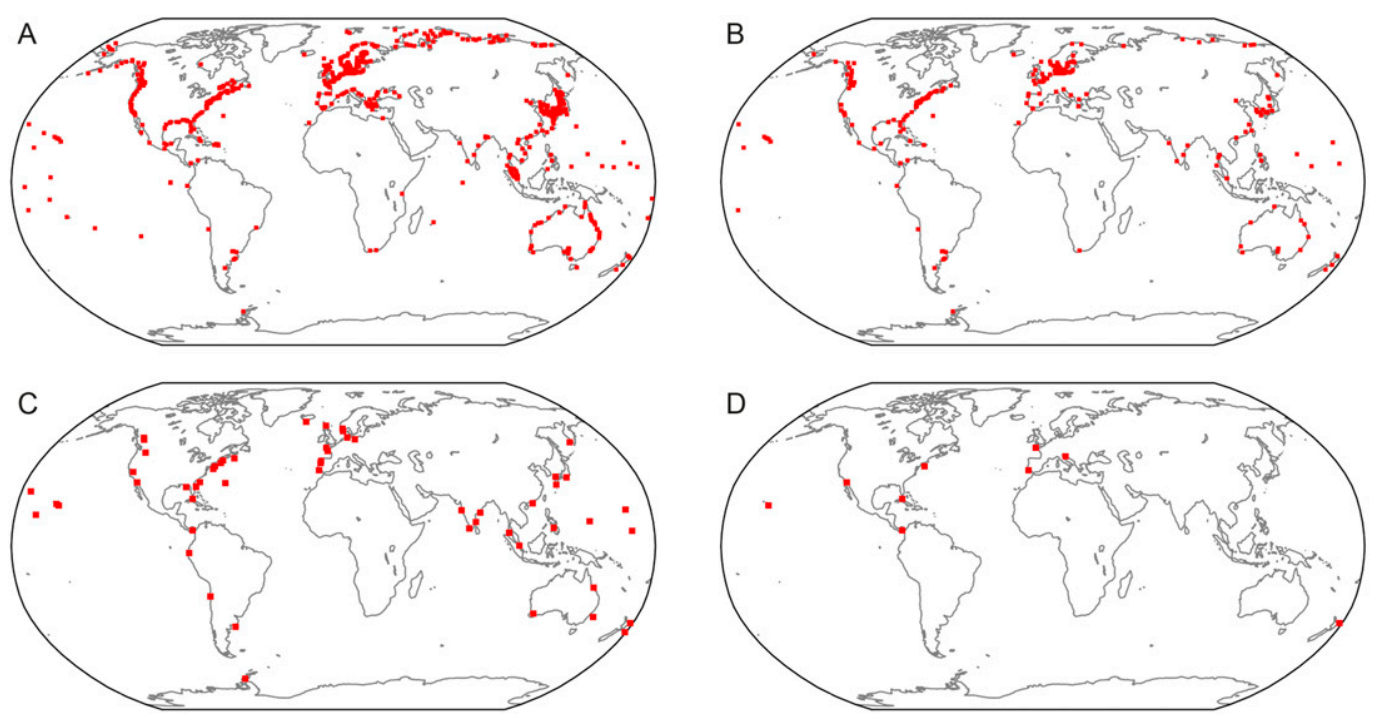

FIG. 1. (a) The locations of the 622 tide gauge sites used by H15. The (b) 224, (c) 56, and (d) 9 overlapping sites between the H15 subset and the subsets of CW2011, RD2011, and Holgate (2007), respectively.

satellite altimeters $\left(\sim \pm 65^{\circ}\right)$. Moreover, these studies favored stations with long, nearly continuous records, and they removed stations with anomalous trends or variability. CW2011 permitted the use of some sites in the PSMSL metric database (raw observations with no common datum requirement) that fall within the altimetry latitudinal range, while RD2011 removed any sites close to regions of known vertical land movement. CW2011 were left with 290 locations (some of which include multiple tide gauges grouped together), while RD2011 employed 89 tide gauges. Holgate (2007) imposed even more stringent criteria. In addition to choosing sites away from areas of known vertical land movement, he required that records had to be $70 \%$ complete between 1904 and 2003, yielding a subset consisting of nine records.

Comparison of the different tide gauges subsets reveals that there are 224,56, and 9 overlapping sites between the H15 subset and the subsets of CW2011, RD2011, and Holgate (2007), respectively (Figs. 1b-d). To test the robustness of the H15 methodology, we ran the multimodel KS using the overlapping sites from these three tide gauge subsets. We use the overlapping sites and not the complete subsets since the overlapping sites are the only RLR sites that meet the KS and GPR criteria of a minimum of $30 \mathrm{yr}$ of data between 1950 and 2010. Our estimates of GMSL obtained in these three tests, overlain with the H15 estimate, are shown in Fig. 2a. While three of the four estimates cover the time window 1880-2010, the analysis time window for the Holgate (2007) subset does not begin until 1900. Our decision to shorten the Holgate (2007) time window is due to limited data availability in the 1800 s for the nine-tide-gauge subset, which can result in a numerically unstable KS. This instability can be derived analytically using the Kalman smoother equations (Gelb et al. 1974). Commencing this analysis in 1900 ensures that the multimodel KS remains stable throughout the entire analysis time window.

Using generalized least squares to determine rates from the posterior probability distributions, we obtain 1901-90 GMSL rates of $1.2 \pm 0.2,1.3 \pm 0.2$, and $1.3 \pm 0.3 \mathrm{~mm} \mathrm{yr}^{-1}$ for the CW2011, RD2011, and Holgate (2007) subsets, respectively (Fig. 2a, inset table). These estimates are consistent with the $\mathrm{H} 15$ estimate of $1.2 \pm 0.2 \mathrm{~mm} \mathrm{yr}^{-1}$ obtained using the larger subset of 622 tide gauge records. While there is greater variability in the rates computed for the period 1993-2010 (Fig. 2a, inset table), the four estimates still agree within their uncertainty ranges. The rates of GMSL change computed using the four different subsets are shown in Fig. 2b. Although there are differences across the four time series, the large-scale features are consistent. In particular, we see a period of higher rates in the 1940s, a decrease in rates in the 1950s, and a gradual long-term increase in rates since the 1960s.

The consistency between the different estimates falsifies the Hamlington and Thompson (2015) hypothesis that the tide gauge selection in H15 introduced a bias in the estimate of GMSL rate of as much as $0.6 \mathrm{~mm} \mathrm{yr}^{-1}$. We conclude that the KS methodology is robust to the range of tide gauge subsets adopted in the literature. This robustness arises from the fact that the methodology extracts global information from the sparse records by modeling the underlying processes responsible for the observed change. 


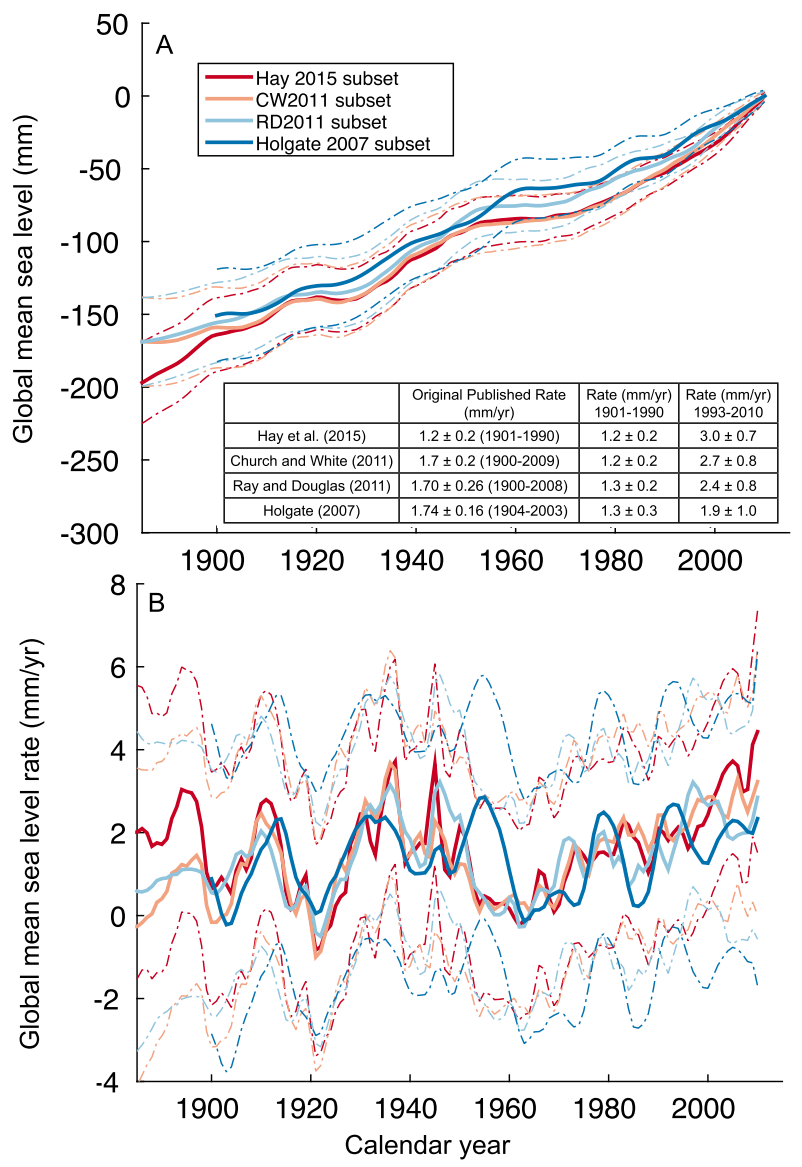

FIG. 2. (a) GMSL time series obtained using the KS applied to various subsets of tide gauges adopted in previous studies: H15 (red line), CW2011 (peach line), RD2011 (light blue line), and Holgate (2007) (dark blue line). The corresponding dashed-dotted lines represent the $\pm 1 \sigma$ uncertainty range. Inset table: original published rates of sea level rise and rates of GMSL change obtained over the time periods 1901-90 and 1993-2010 for each of the KS analyses. The uncertainties listed in the table represent the $90 \% \mathrm{CI}$ (b) Time series of annual GMSL rates for the four subsets presented in (a). All results are presented beginning in 1880 except for the Holgate (2007) subset, which starts in 1900 (see text).

\section{b. Random tide gauge selection}

The robustness of the KS methodology to tide gauge selection must have its limits. The above subsets were chosen so that they sample multiple ocean basins, include tide gauges with minimal vertical land movement, and do not contain sites with anomalous trends. The resulting subsets contain what the respective authors deemed to be the best tide gauges to use in estimating GMSL. One would expect that the estimate, and its uncertainty, would be substantially different if one adopted, for example, a handful of tide gauge records from sites in the same region. To explore this issue, we have performed an additional sensitivity test.
As discussed above, the KS methodology incorporates a multimodel component associated with a suite of simulations of the sea level signal due to GIA and ocean dynamics. In the following test, in order to reduce computational requirements, we set the GIA model to the ICE-5G ice history and a mantle viscosity profile close to the VM2 model (Peltier 2004) and the ocean model to the multimodel estimate derived by H15. As a baseline, we ran the KS and the Kalman filter (KF; which represents the forward path in the forward-backward Kalman smoother methodology) using the above GIA-ocean dynamics model combination for the full set of 622 sites. We obtained 1901-90 GMSL rates of rise of $1.3 \pm 0.2$ and $1.1 \pm$ $0.3 \mathrm{~mm} \mathrm{yr}^{-1}$ for the KS and KF, respectively. The KF rate is expected to be different (and its uncertainty higher) than the estimate obtained using the KS because the KF's annual estimate of GMSL is based solely on tide gauge observations prior to and including the analysis year, whereas the KS includes information from the full 1880-2010 time range. More specifically, in the case of increasing rates of sea level rise, the KF estimate will be biased low. However, in a world of decreasing rates of sea level rise, the KF GMSL estimate would be biased high.

The KF was then run 10000 times for randomly chosen subsets comprising $10,50,100,200$, or 500 tide gauges. As noted above, the KS can become unstable in the absence of observations, a situation that can easily occur when randomly choosing a small number of tide gauge records (e.g., 10) with their associated data gaps. We removed the smoothing component of the KS approach in order to avoid this instability. This initial set of 10000 runs had a prior GMSL rate estimate of $0 \mathrm{~mm} \mathrm{yr}^{-1}$ with a standard deviation of $10 \mathrm{~mm} \mathrm{yr}^{-1}$.

We binned the 10000 GMSL estimates on the basis of the number of tide gauges used in the analysis, and in each case we computed the mean and standard deviation of the resulting distribution. Figure 3 shows the mean and standard deviation as a function of the number of gauges (blue line). These results indicate, as expected, that the spread in estimates of the rate of rise narrows significantly as you increase the number of tide gauge records included in the analysis. In the case of 10 randomly selected tide gauge records, the standard deviation of the suite of estimates is approximately $0.5 \mathrm{~mm} \mathrm{yr}^{-1}$ (with minimum and maximum values of -2.2 and $2.7 \mathrm{~mm} \mathrm{yr}^{-1}$, respectively), and this spread decreases to approximately $0.2 \mathrm{~mm} \mathrm{yr}^{-1}$ in the case of 200 gauges. We also note that the mean GMSL rate estimated in each of these various bins converges to the estimate from the 622 tide gauge run discussed above $\left(1.1 \mathrm{~mm} \mathrm{yr}^{-1}\right)$ once the random sampling involves 500 tide gauges. These results indicate that the criteria used in previous studies (Holgate 2007; CW2011; RD2011), 


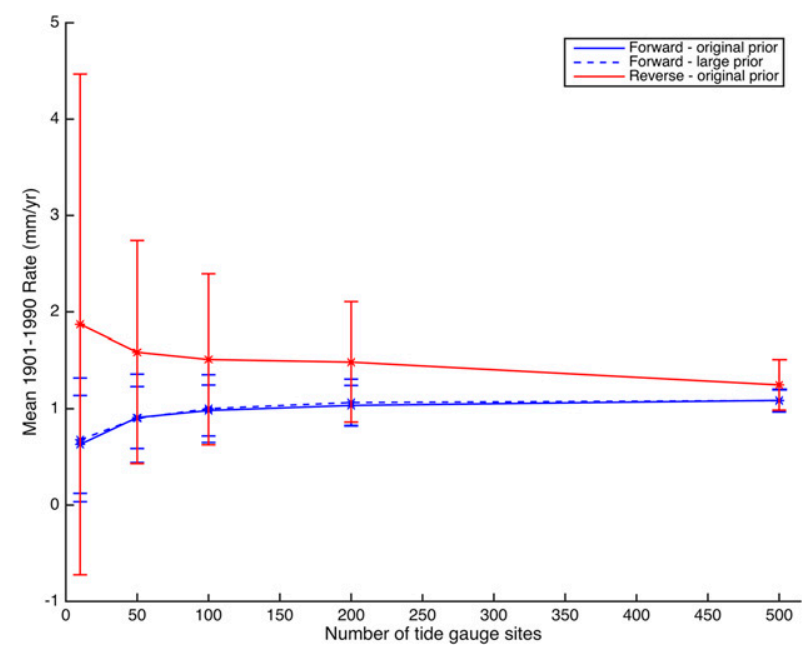

FIG. 3. Average GMSL rate and std dev of the distribution of 1901-90 GMSL rate estimates as a function of the number of randomly chosen subsets of tide gauges in each distribution. The estimates are based on a KF methodology with one GIA-ocean dynamics model combination (see text). The results in blue adopt one of two prior models: a GMSL rate of $0 \mathrm{~mm} \mathrm{yr}^{-1}$ for each of the 22 sources with a std dev of $10 \mathrm{~mm} \mathrm{yr}^{-1}$ (solid line) or a GMSL rate of $0.5 \mathrm{~mm} \mathrm{yr}^{-1}$ for each source with a std dev of $100 \mathrm{~mm} \mathrm{yr}^{-1}$ (dashed line). In each case, a total of 10000 subsets of tide gauges were selected to guarantee converged distributions. The red curve shows the results when the KF is run backward in time from 2010 to 1880 with a prior GMSL rate (in 2010) of $1 \mathrm{~mm} \mathrm{yr}^{-1}$ with a std dev of $10 \mathrm{~mm} \mathrm{yr}^{-1}$ for each of the 22 sources.

which lead to consistent KS-based estimates of the GMSL rate, provide more informative estimates than a random sampling of tide gauge sites.

We repeated the full, 10000-run procedure a second time starting from a prior model with melt rates equal to $0.5 \mathrm{~mm} \mathrm{yr}^{-1}$ for each of the 22 sources (i.e., $11 \mathrm{~mm} \mathrm{yr}^{-1}$ for the total GMSL rate) and standard deviation of $100 \mathrm{~mm} \mathrm{yr}^{-1}$. The results once again show reduced spread as the number of randomly selected tide gauge sites increases (Fig. 3, blue dashed line). Furthermore, as before, the mean of the distributions converged to the estimate obtained using 622 tide gauges $\left(1.1 \mathrm{~mm} \mathrm{yr}^{-1}\right)$ once the sampling included 500 tide gauges.

The fact that the final distributions in these two, 10000 simulation Monte Carlo tests converged to the same value indicates that the prior model for the GMSL rate in the analysis was noninformative. It is important to establish the same fact for the full KS analysis discussed in H15 (Fig. 2, blue line). That analysis adopted a prior estimate for the GMSL rate of $0 \mathrm{~mm} \mathrm{yr}^{-1}$ with a standard deviation of $10 \mathrm{~mm} \mathrm{yr}^{-1}$ for each of the 22 sources to obtain a final GMSL estimate of $1.21 \pm 0.20 \mathrm{~mm} \mathrm{yr}^{-1}$. We have repeated that analysis using a prior estimate for the GMSL rate of $0.5 \mathrm{~mm} \mathrm{yr}^{-1}$ for each of the 22 sources with a standard deviation of $100 \mathrm{~mm} \mathrm{yr}^{-1}$. The estimated (total) GMSL rate was $1.22 \pm 0.20 \mathrm{~mm} \mathrm{yr}^{-1}$, confirming once again that the prior was noninformative.

To demonstrate the effect on GMSL estimates of only running the $\mathrm{KF}$ in one direction, we repeated the same Monte Carlo tests described above but for the KF run in reverse (i.e., backward in time from 2010 through 1880). In these tests, we initialized the prior model in 2010 with melt rates of $0.05 \mathrm{~mm} \mathrm{yr}^{-1}$ and a standard deviation of $10 \mathrm{~mm} \mathrm{yr}^{-1}$ for each of the 22 sources. The results (Fig. 3, red line) clearly demonstrate that in the case of decreasing rates of sea level rise, KF-based estimates of GMSL will be biased high, particularly for a small subset of tide gauges. (In the case of 10 randomly selected tide gauge records, the standard deviation of the estimates is approximately $2.6 \mathrm{~mm} \mathrm{yr}^{-1}$, with minimum and maximum values of -20.2 and $18.3 \mathrm{~mm} \mathrm{yr}^{-1}$, respectively.) To eliminate these potential biases, completing both the forward and backward passes of the Kalman smoother for a large subset of tide gauges, as was done in the H15 analysis, is necessary.

\section{Resolvability of individual melt rates}

\section{a. Synthetic tests}

Although the total GMSL rate (i.e., the sum of the individual contributions) is consistent for the various tide gauge subsets considered in section $3 \mathrm{a}$, we next test whether this insensitivity extends to estimates of individual melt rates. To explore this issue, we perform a series of tests on synthetic records. These tests highlight an important point regarding the separability of the individual estimates.

To begin, we created a simple synthetic global sea level field for the years 1880-2000 with three contributions. The first is a signal from Greenland Ice Sheet (GIS) mass loss. The fingerprint of this nonuniform signal (Fig. 4, top) is characterized by an extensive region of sea level fall close to the ice margin and sea level rise in the far-field regions. The second contributor is a globally uniform signal, and the third is spatially uncorrelated white noise with a global mean value of $0.0 \mathrm{~mm}$ and a standard deviation of $2 \mathrm{~mm}$. The time series of GMSL and the two contributing signals are shown as the red curves in Figs. 4a-c. In this test, we sample the global sea level field at two locations and run the GPR methodology (H15) over the analysis time window. In every decade, we estimate the GMSL contributions from both sources, as well as their sum. The GPR estimate and its one standard deviation $(1 \sigma)$ uncertainty are shown by the solid and dashed blue lines, respectively, on each frame.

In the first test, the two sites are located in the far field of the GIS (orange-filled circles in Fig. 4, top). The results (Figs. 4a-c) indicate that when the observations do not capture the spatial variability in the fingerprint, the ability 

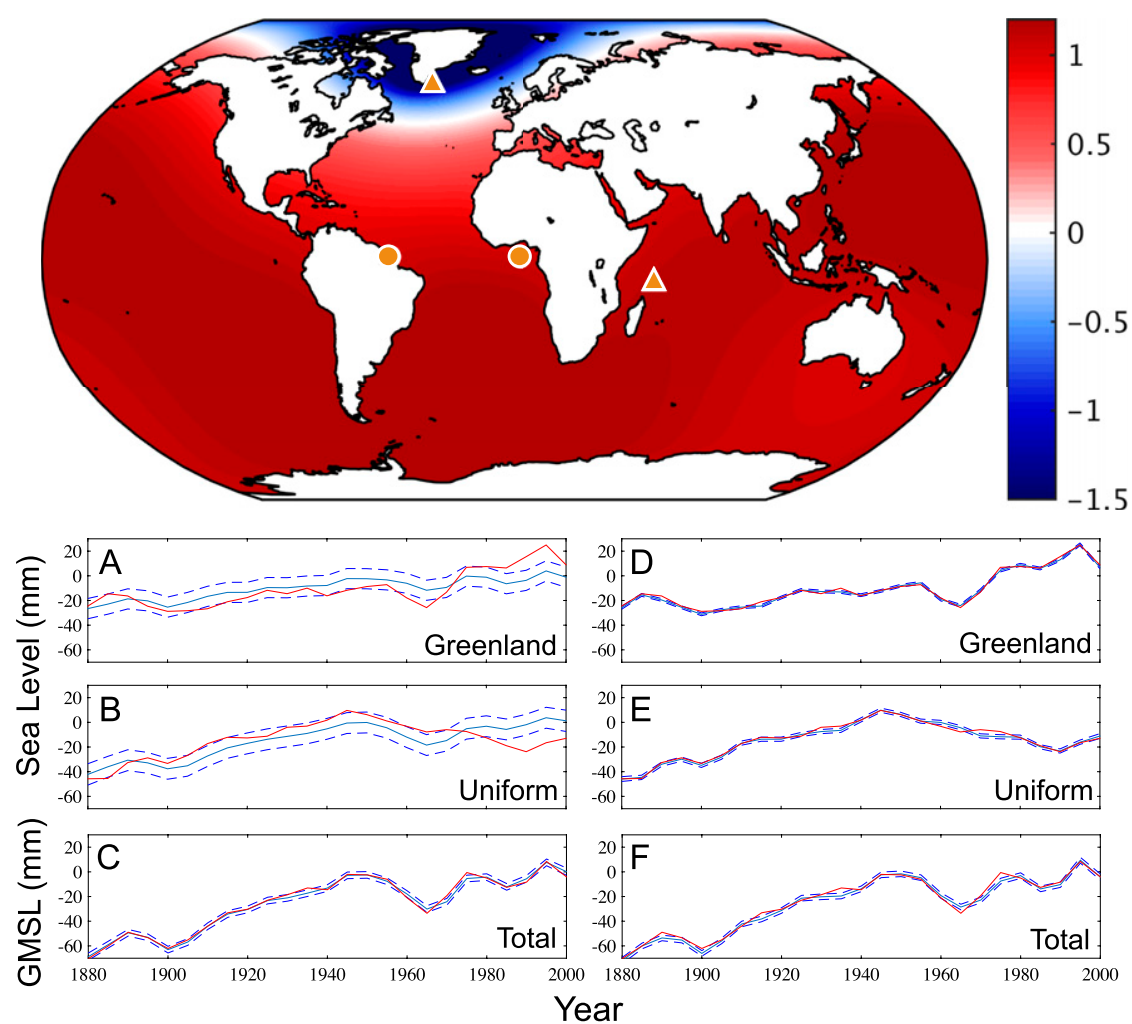

FIG. 4. (top) Normalized relative sea level fingerprint for instantaneous melting from the GIS (ratio of local to global mean sea level rise). Also shown are the locations of the sites used in the synthetic far-field-only (orange-filled circles) and near-field-plus-far-field (orange-filled triangles) sensitivity tests. Results of the (a)-(c) far-field-only and (d)-(f) near-field-plus-farfield synthetic tests (see text) are shown. The red curves are the true sea level signals input into the synthetic calculation, while the blue lines are the estimated contributions (solid) and their $1 \sigma$ uncertainty ranges (dashed): (a),(d) GIS contributions; (b),(e) the uniform sea level signals; and (c),(f) the total signals (i.e., the sum of the individual contributions).

to independently estimate the two sea level contributions is limited. Large uncertainties are apparent in the estimates of both time series, the correlation coefficient between the two estimates is -0.95 , and the $1 \sigma$ uncertainty ranges do not always encompass the true values (Figs. 4a,b). However, while the individual contributions are not well resolved, the sum of the two components (Fig. 4c) is precisely estimated. In the second test, one site is located in the near field of the GIS and one is located in the far field (orange triangles in Fig. 4, top). In this case, both the individual contributions (Figs. 4d,e) and their sum (Fig. 4f) accurately track the true values, and the correlation coefficient between the two estimates decreases to 0.25 . These simple tests indicate that a robust separation of individual contributions to GMSL requires that the observations sample the full spatial variability of the sea level field.

Extending this simple synthetic test to include melt from all 22 ice sheets and mountain glaciers considered in H15, we construct synthetic sea level time series for a global $5^{\circ} \times 5^{\circ}$ grid, imposing constant melt rates over the analysis time window such that the total rate of GMSL rise is $1.1 \mathrm{~mm} \mathrm{yr}^{-1}$ (Table 1). In addition to the melt signals, the sea level time series also include temporally uncorrelated noise with a spatial covariance described by an exponential decay function with a decay length scale of $1500 \mathrm{~km}$ and a standard deviation of $1 \mathrm{~cm}$. Using the synthetic sea level data, we perform two tests. For the first test, we run the KS using the full global grid of observations. In the second test, we sample the global grid at the tide gauge sites and impose the data availability that is present in the PSMSL tide gauge observations. For the second test, we also include spatially correlated noise by adding the H15 estimated ocean dynamics signal to the synthetic time series while also removing the ocean model component of the control input parameter in Eq. (1). By removing this component of the filter, we are adding regional sea level changes to our synthetics that we do not explicitly model or account for in the Kalman smoother.

The results of these two tests reveal that a global set of observations allows for robust inference of individual 
TABLE 1. Rates used as input for the synthetic sea level test with 22 melt sources.

\begin{tabular}{lc}
\hline \multicolumn{1}{c}{ Melt source } & Synthetic truth $\left(\mathrm{mm} \mathrm{yr}^{-1}\right)$ \\
\hline Uniform & 0.42 \\
GIS & 0.14 \\
WAIS & 0.10 \\
East Antarctic Ice Sheet (EAIS) & 0.005 \\
Iceland & 0.031 \\
Svalbard & 0.008 \\
Novaya Zemlya & 0.011 \\
Severnaya Zemlya & 0.003 \\
Kamchatka & 0.006 \\
High-Mountain Asia (HMA) 1 & 0.011 \\
HMA 2 & 0.008 \\
Caucasus & -0.003 \\
Alps & 0.006 \\
Scandinavia & -0.008 \\
Alaska & 0.123 \\
Western Canada & -0.014 \\
Baffin Island & 0.093 \\
Ellesmere Island & 0.094 \\
Low-latitude Andes & 0.017 \\
Patagonia & 0.064 \\
New Zealand & -0.006 \\
Franz Josef Land & 0 \\
GMSL & 1.12 \\
\hline
\end{tabular}

melt sources. Specifically, we estimate GIS and West Antarctic Ice Sheet (WAIS) melt rates of $0.14 \pm 0.02$ and $0.11 \pm 0.02 \mathrm{~mm} \mathrm{yr}^{-1}[90 \%$ confidence interval (CI)], respectively, and a uniform sea level rise of $0.42 \pm$ $0.1 \mathrm{~mm} \mathrm{yr}^{-1}$. The remaining 19 sources show similar agreement with the rates input into the synthetic calculation. However, when the sea level data are restricted to the PSMSL data availability, the individual melt estimates misfit the rates associated with the synthetic calculation, along with their uncertainties, increase significantly. As an example, we estimate GIS, WAIS, and uniform contributions of $-0.06 \pm 0.09,0.24 \pm 0.06$, and $0.2 \pm 0.2 \mathrm{~mm} \mathrm{yr}^{-1}$, respectively. We conclude that the tide gauge sampling of the synthetic sea level field is incapable of rigorously separating the sources of mass flux included in the synthetic dataset. However, while the individual contributions are subject to significant uncertainty, the sum of all 22 sources is robustly inferred. For the case of both global observations and PSMSL tide gauge sampling, we estimate the synthetic GMSL rates of rise to be $1.1 \pm 0.1 \mathrm{~mm} \mathrm{yr}^{-1}$, in agreement with the total melt incorporated into the synthetic calculation.

\section{b. The H15 analysis}

As is clear from the above discussion, the ability to uniquely identify the amplitude of a particular melt source is a function of the proximity of the observations to the ice sheet or glacier. Determining the resolvability of model parameters or, more generally, any weighted combination of model parameters within a Bayesian analysis involves a comparison of the prior and posterior variances (Backus 1988). In particular, any set of different model parameter combinations is resolvable if all such combinations are uncorrelated (or only weakly correlated) and if the posterior variance of the combination is significantly smaller than the prior variance.

A powerful method of finding resolvable combinations of model parameters involves the precision matrix (i.e., inverse covariance matrix) of the measurements $\mathbf{V}_{m}^{-1}$, computed as the difference between the posterior and prior precision matrices:

$$
\mathbf{V}_{m}^{-1}=\mathbf{V}_{\mathrm{po}}^{-1}-\mathbf{V}_{\mathrm{pr}}^{-1}
$$

where $\mathbf{V}_{\mathrm{pr}}$ and $\mathbf{V}_{\mathrm{po}}$ are the prior and posterior covariance matrices of the model estimates, respectively. Since all the probability distributions are assumed to be multivariate Gaussian distributions, zero-valued elements in the precision matrix indicate a conditional independence between parameters, while a nonzero off-diagonal element indicates that a partial correlation exists between the associated pair of model parameters. However, in the case when individual parameters cannot be resolved, it may still be possible that linear combinations of the estimated parameters are uncorrelated and resolvable.

The uncorrelated linear combinations of the estimated model parameters are given by the eigenvectors of the measurement precision matrix $\mathbf{V}_{m}^{-1}$ [Eq. (9)], and thus the number of such combinations is equal to the number of parameters being estimated. In the $\mathrm{H} 15$ analysis, there are 22 such combinations. To determine the number of these combinations $N$ that can be resolved, that is, the number of 22 combinations whose posterior variance is significantly smaller than the prior variance $\left(V_{\mathrm{po}} / V_{\mathrm{pr}} \ll 1\right)$, we compute $N$ as the trace of the resolution matrix $\mathbf{R}$ (Backus 1988):

$$
\begin{aligned}
& \mathbf{R}=\mathbf{V}_{\mathrm{po}} \mathbf{V}_{m}^{-1} \text { and } \\
& N=\operatorname{tr}(\mathbf{R}) .
\end{aligned}
$$

The $N$ resolvable combinations of model parameters will have the largest variance reduction. In the ideal case, $N$ will be close to the total number of model parameters and all $N$ combinations will be strongly weighted toward the estimate of a single parameter (i.e., the weights that define the combinations will be close to zero for all but one parameter). In any practical application, this will not be the case, but the combinations that are resolved may have a reasonably clear physical interpretation (Backus 1988).

As a simple illustration of this concept, we first return to our two-source, two-site synthetic test. Figure 5 shows the weightings of the two parameters (GIS melt and 

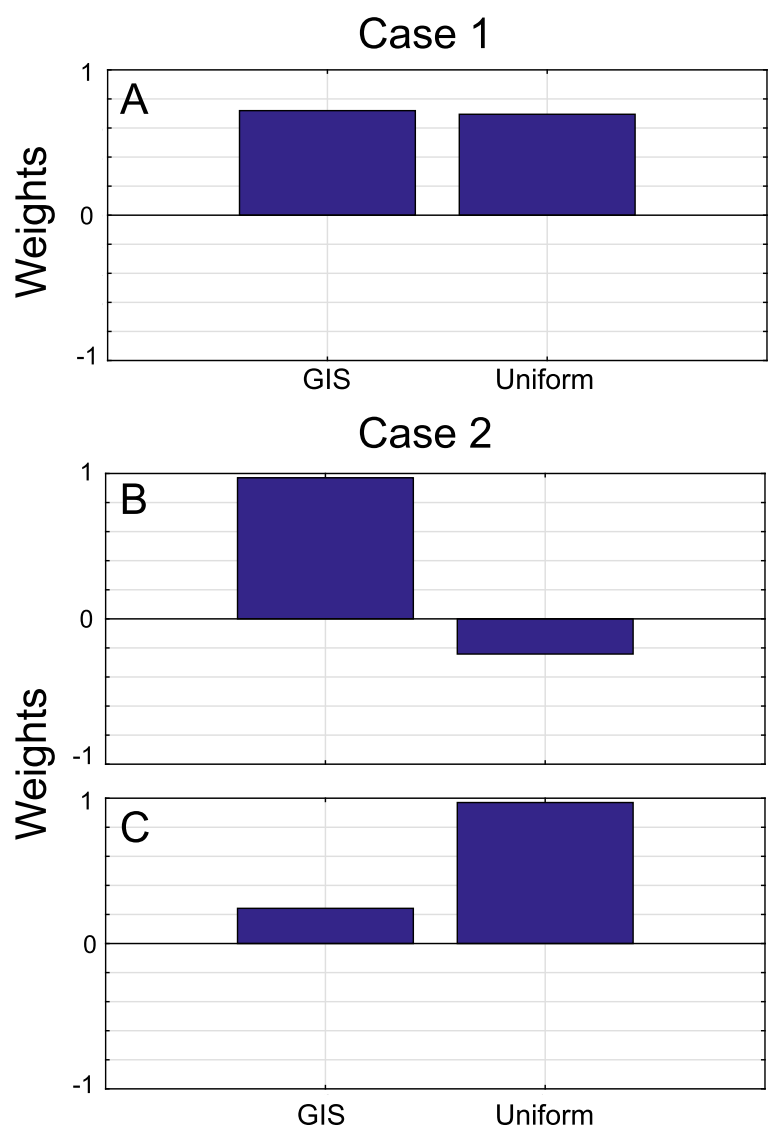

FIG. 5. Weights in the combinations of the two model parameters in the two-source synthetic tests that are resolved by the observations (see text). (a) The weights associated with the analysis with two far-field observations (case 1); (b),(c) analogous results for the analysis in which an observation is included from both the far field and near field of the melt center (case 2).

uniform signal) associated with the near-field-only and far-field-plus-near-field data analyses. The far-field-only case (case 1) has one resolvable combination of parameters $(N=1.03)$ characterized by roughly equal weightings between the two model parameters (Fig. 5a). This is a quantitative demonstration that the two model parameters cannot be independently estimated when the observations come solely from the far field of the melt event. As we have noted, the strong correlation between the two estimates manifests itself as large uncertainties in the estimates of the individual parameters (Figs. 4a,b) and as a small uncertainty in their sum (Fig. 4c). In contrast, the case with observations in both the near field and far field (case 2) has two resolvable combinations $(N=1.99)$. Figures $5 \mathrm{~b}$ and $5 \mathrm{c}$ show the two linear combinations of model parameters that are resolved in the analysis, each of which is dominated by the estimate of one of the two model parameters. Thus, as we demonstrated above, the two individual source contributions can be estimated with good precision.

With this insight in hand, we now apply the same methodology to the KS results of H15. In the case of the estimates for the period 1901-90, we find that the trace of the resolution matrix $(N=4.3)$ indicates that there are approximately four linear combinations of the 22 model parameters that are uncorrelated with each other and that are resolvable (i.e., the posterior variance of the combination is much smaller than the prior variance). Each frame in Fig. 6 shows the weightings associated with one of the resolvable combinations. The dominant sources are labeled in each case, and the variance reductions associated with the weighted sums are provided in each frame. The first combination in Fig. 6 (i.e., the weighted sum with the largest variance reduction) is composed of small to moderate contributions from most of the 22 melt sources. The next two resolvable combinations (Figs. 6b,c) have major contributions from estimates of glacier flux in Svalbard and Franz Josef Land and small contributions from a number of other glaciers. While the estimated GIS mass flux is the dominant source in the final resolvable combination (Fig. 6d), this combination is characterized by significant contributions from most of the other sources of GMSL.

\section{Global sea level fields}

In addition to providing estimates of mass balance, GMSL, and sea level reconstructions at tide gauge sites, both the KS and GPR methodologies estimate the full spatiotemporal sea level field. From this field we have computed maps of the rates of sea level rise in the period 1901-90 for both statistical methodologies (Figs. 7a,c) and their $1 \sigma$ uncertainty (Figs. 7d,f). Figures 7a,c include sea level changes due to GIA, contemporary land ice melting, thermal expansion, a uniform sea level term, and local ocean dynamics, and thus the spatial variability apparent in the maps is a reflection of the geometries that characterize these processes. Overlaid on Fig. 7 are linear rates of sea level change estimated using the observations at 85 tide gauge sites with at least $60 \mathrm{yr}$ of data over 1901-90. There is good agreement between the tide gauge rates and the local grid points, indicating that both methodologies are reproducing the local features that are present in the observations. Scatterplots comparing observed and estimated linear rates across this 90-yr time interval (as well as across the period 1993-2010) are shown in Figs. S1-S4 of the supplemental material.

There are two prominent differences between the KS and GPR reconstructions. In data-poor regions, such as midocean basins, there are few observations to constrain the reconstruction, particularly the geographic sea level 


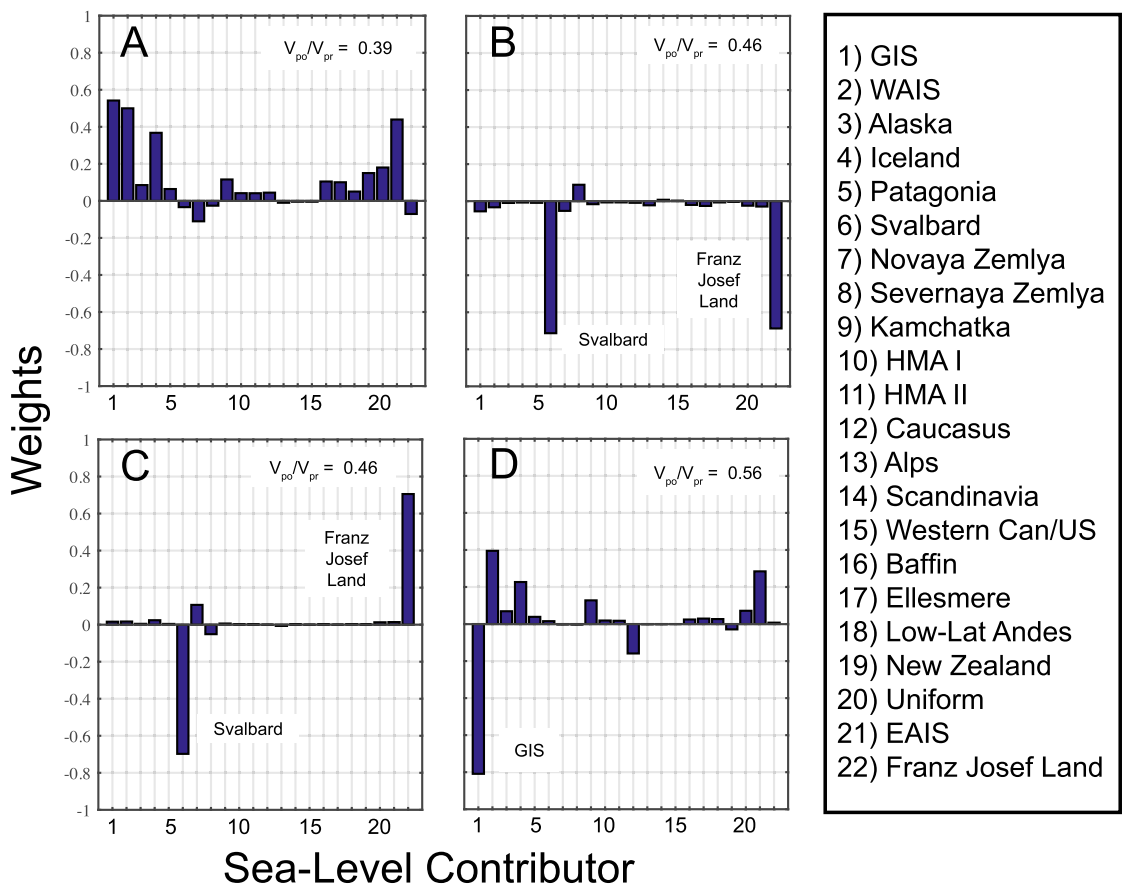

FIG. 6. Weightings that define the four linear combinations of model parameters resolvable in the KS analysis of $\mathrm{H} 15$ for the 1901-90 time period. Also included in each panel are the ratios of the posterior and prior variances for the weighted sums. The dominant contributors in each frame are labeled.

variability due to ocean dynamics. This is evident in the large uncertainties in Figs. 7d,f. In the KS analysis, the geographic variability in the signal due to ocean dynamics is constrained using the weighted mean of a set of CMIP5 climate simulations (H15), and these may be limited in their ability to correctly model small-scale oceanic features (Flato et al. 2013). In the GPR analysis, the ocean dynamic signal is captured in two different terms: the first involves the unweighted mean and covariance of the distribution of the CMIP5 models, and the second is a Matérn covariance function. Since the unweighted mean of the ocean model simulations is a smoother field than the weighted mean, the sea level rates in the middle of the ocean basins estimated in the GPR analysis show less variability than the KS-derived result.

The second prominent difference between the two global reconstructions is the spatial scale of local features that can be observed at some tide gauge sites (e.g., sea level changes at sites in Japan, an area with active tectonics, and in the Gulf of Mexico, where fluid withdrawal and sediment compaction are high). The decorrelation length scale prescribed in the GPR methodology is smaller than the equivalent length scale in the KS method, and this results in the "smearing" of local signals in the KS reconstruction. As an example, in the rate map derived using the KS methodology (Fig. 7a), the local tectonic signal observed at tide gauges in Japan spreads into the adjacent Pacific Ocean. The equivalent "tectonic footprint" is much smaller (close to pixel size) in the GPR-derived rate map (Fig. 7c). In the GPR methodology, the Matérn function captures small-scale sea level signals due to local tectonics, sediment compaction, and ocean dynamics. In contrast, there is no term in the KS method that explicitly estimates these local signals. The KS is unable to partition these regional sea level signals into the modeled processes (i.e., melt, GIA, and ocean dynamics), and they become part of the filter process noise.

In the KS, the estimate of the global field is not directly informed by the tide gauge observations. Instead, the global field is treated as a latent variable with sea level rates solely informed by the estimated melt rates. The effect of the KS being formulated this way is that the uncertainties on the global fields remain large over the entire analysis time window (Fig. 7d). Reformulating the KS to directly connect the global grid to the tide gauge sites will be an important next step. We note that while the rates of sea level change on our KS global grid are not statistically distinguishable from zero in most areas of the ocean, there are two important exceptions. The first is at the tide gauges themselves (i.e., locations with observations), and the second is in regions with large rates of sea level change (e.g., areas that are dominated 
A

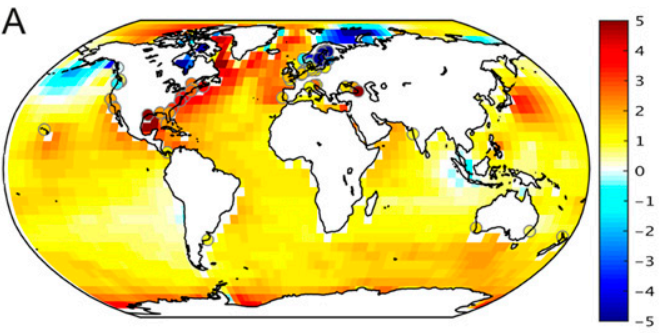

$\mathrm{B}$
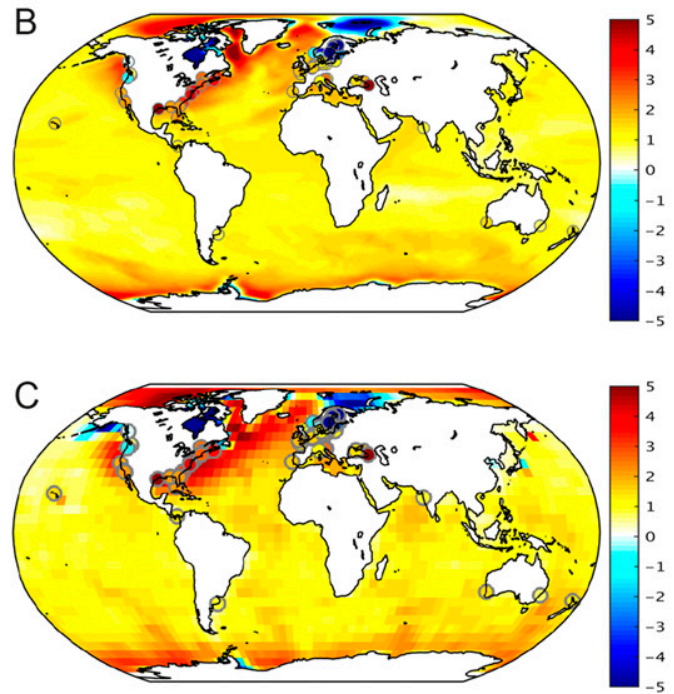
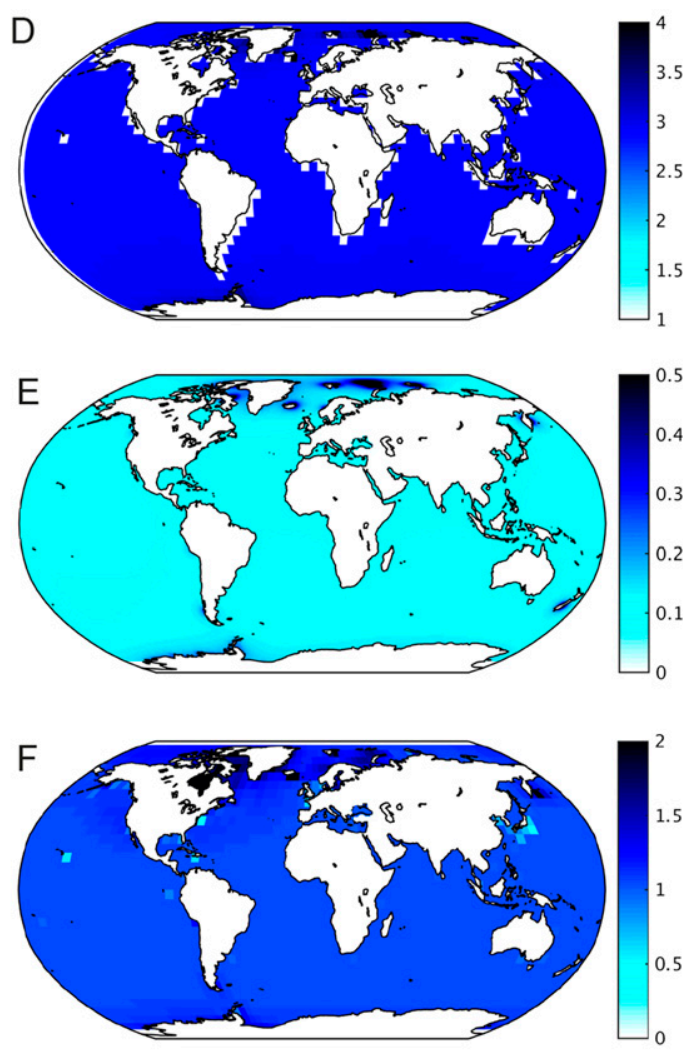

FIG. 7. Spatial maps of sea level change for 1901-90 ( $\left.\mathrm{mm} \mathrm{yr}^{-1}\right)$ estimated using the (a) KS methodology; (b) the estimated KS melt rates, ocean dynamics, and GIA fields; and (c) the GPR methodology. These maps include sea level changes due to GIA, contemporary land ice melting, uniform thermal expansion, and ocean dynamics. Overlaid are rates of change estimated at tide gauges with at least $60 \mathrm{yr}$ of data within the time range 1901-90. (d)-(f) As in (a)-(c), but for the $1 \sigma$ uncertainty grids. The KS fields in (a),(d) are computed by fixing the GIA and ocean dynamics models in the KS to the multimodel estimates of H15.

by postglacial rebound). We also emphasize that GMSL is computed by summing the source contributions and not by computing the weighted sum of the global grid.

An alternative approach to reconstructing the global sea level field is to use the KS posterior estimates of land ice melt (and their respective sea level fingerprints), uniform thermal expansion, GIA, and ocean dynamics. We emphasize that this field (Fig. 7b), along with its associated uncertainty (Fig. 7e), includes only the melt, GIA, and ocean dynamics sea level changes that are included in the KS estimate of GMSL. Local sea level changes that are present in the tide gauge observations and in the KSderived global sea level field (e.g., changes due to tectonics and sediment compaction) are not included in this reconstruction. The resulting uncertainties in this posterior estimate (Fig. 7e) are significantly smaller than the uncertainties obtained for the KS-derived field (Fig. 7d).

Common to all three reconstructions are the large uncertainties in the rates (Figs. $7 \mathrm{~d}-\mathrm{f}$ ) in areas close to land-ice reservoirs, such as Greenland, Iceland, and the glaciers of northern Canada. Large uncertainties in the individual melt contributions, which reflect our inability to separate the individual melt contributions, propagate into our sea level rise estimates and produce the large uncertainties estimated in these regions.

Calculating the difference between the sea level field estimated within the KS (i.e., Fig. 7a) and the reconstructed KS posterior field (i.e., Fig. 7b) produces a map of the KS residual regional sea level not described by the melt, GIA, uniform thermal expansion, and ocean dynamics processes (Fig. 8). The areas with large signals in Fig. 8 are the same regions noted above (i.e., these areas involve localized signals that have been smeared into broader areas). These signals do not contribute to the estimates of GMSL; the latter includes only melt and ocean thermal expansion contributions.

Over the shorter time period 1993-2010, decadal and interannual variability in the CMIP5 ocean models strongly impacts the estimated spatial patterns in the ocean dynamics signal, and this makes the estimate sensitive to the preferred ocean model. Any uncertainties in the ocean model will therefore dominate the regional 


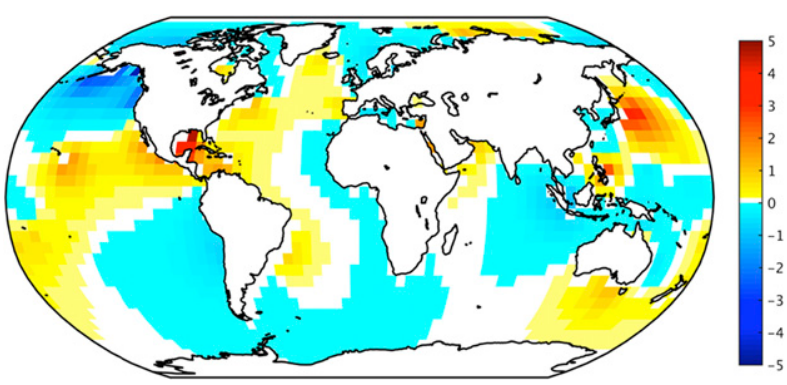

FIG. 8. KS residual regional sea level change $\left(\mathrm{mm} \mathrm{yr}^{-1}\right)$ for $1901-90$ (see text).

signals. Without additional observations constraining the sea level field in data-poor regions, regional variability in the rates cannot be robustly determined over this time period using the KS and GPR methodologies.

\section{Conclusions}

The rates of GMSL change estimated by $\mathrm{H} 15$ for the period $1901-90$ are about $20 \%-30 \%$ lower than previous estimates obtained using regional averaging or EOFbased approaches. The new analysis has faced two types of criticism. First, it has been argued that the lowered estimate of 1901-90 GMSL change in H15 is due to a less stringent selection of tide gauge observations and in particular the inclusion of records that are contaminated by local processes (Hamlington and Thompson 2015). Second, the H15 methodologies yield estimates of individual contributions that have relatively large uncertainty, and it has been suggested that this is inconsistent with the more precise estimate of total GMSL rate quoted in the study.

To address the first, we performed two experiments that explored the sensitivity of the KS approach to tide gauge selection. To begin, we adopted various sets of tide gauges favored in previous studies on the basis of record length, tectonic stability, etc. and found that the resulting KS-based estimates of the GMSL rate were statistically unchanged from the $\mathrm{H} 15$ estimate of $1.2 \pm 0.2 \mathrm{~mm} \mathrm{yr}^{-1}$. This is a direct test of the argument in Hamlington and Thompson (2015), and on this basis their argument can be rejected. Next, using different subset sizes, we chose tide gauges at random and applied the forward filter component of the KS methodology to estimate GMSL. We repeated the procedure 10000 times for each subset size and for two different priors, and found, as expected, that as the number of tide gauge sites increased, the width of the distribution of computed GMSL rates decreased significantly and the mean of the distribution converged to the value obtained using the 622 tide gauge records adopted in $\mathrm{H} 15$, approximately $1.1 \mathrm{~mm} \mathrm{yr}^{-1}$.

The potential for any analysis to resolve the individual contributions to GMSL is fundamentally constrained by the information content of the available tide gauge observations, not by the methodology used to estimate GMSL. Posterior correlations between estimates of the individual sources, which arise from incomplete information, can map into large errors in these estimates. However, this basic statistical issue does not necessarily imply that the sum of the model parameters is poorly constrained. This point was highlighted in the first application of the fingerprinting methodology to tide gauge data (Mitrovica et al. 2001), and we have illustrated it herein using three synthetic melt scenarios. Moreover, we have also shown that in the real, 22 -source KS analysis, the incompleteness in both space and time of the tide gauge records leads to strong correlations among individual source estimates. A thorough analysis of the posterior covariance matrix, similar to the one performed here, is essential if one wishes to obtain statistically meaningful estimates of individual melt rates or to identify combinations of the 22 estimates that are resolved by the data (i.e., sets of combinations that are uncorrelated and characterized by a posterior variance significantly smaller than the prior variance). Although individual melt rates over the period 1901-90 are difficult to resolve, a sum of these estimates that accounts for the full posterior model covariances (i.e., the total GMSL rate for 190190) is nevertheless precisely determined.

The results in section $4 \mathrm{~b}$ represent the best-case scenario for the resolvability of estimates of GMSL contributions over the period 1901-90 based on tide gauge records. After about 1990, the number of Arctic tide gauges significantly decreased, and this degrades the resolving power of estimates for the last two decades. However, the availability of satellite altimetry measurements of sea surface height changes in the last two decades will counter this loss of information, and in future work we will explore the resolving power of the complete (tide gauge plus altimeter) dataset in estimating the various contributions to GMSL.

In addition to providing robust estimates of GMSL, both Bayesian methodologies yield estimates of timevarying sea level at tide gauge sites and at locations with no observations. With their ability to accommodate nonstationary spatiotemporal models of sea level, the KS- and GPR-based global sea level reconstructions through the twentieth century improve upon previous studies that relied on stationarity for the reconstructions while also incorporating spatial covariance via process-based sea level modeling. Our global reconstructions, including the maps of sea level rates in Fig. 7, agree well with observations, where they exist, and allow us to examine sea level in data-sparse regions. 
Acknowledgments. This work was supported by the U.S. National Science Foundation (Grants ARC-1203414, ARC1203415, and OCE-1458904) and Harvard University.

\section{REFERENCES}

Backus, G. E., 1988: Bayesian inference in geomagnetism. Geophys. J. Int., 92, 125-142, doi:10.1111/j.1365-246X.1988.tb01127.x.

Blom, H., and Y. Bar-Shalom, 1988: The interacting multiple model algorithm for systems with Markovian switching coefficients. IEEE Trans. Automat. Contr., 33, 780-783, doi:10.1109/9.1299.

Calafat, F. M., D. P. Chambers, and M. N. Tsimplis, 2014: On the ability of global sea level reconstructions to determine trends and variability. J. Geophys. Res. Oceans, 119, 1572-1592, doi:10.1002/2013JC009298.

Cazenave, A., and W. Llovel, 2010: Contemporary sea level rise. Аnпu. Rev. Mar. Sci., 2, 145-173, doi:10.1146/ annurev-marine-120308-081105.

Christiansen, B., T. Schmith, and P. Thejll, 2010: A surrogate ensemble study of sea level reconstructions. J. Climate, 23, 43064326, doi:10.1175/2010JCLI3014.1.

Church, J., and N. J. White, 2006: A 20th century acceleration in global sea-level rise. Geophys. Res. Lett., 33, L01602, doi:10.1029/2005GL024826.

— and - 2011: Sea-level rise from the late 19th to the early 21st century. Surv. Geophys., 32, 585-602, doi:10.1007/ s10712-011-9119-1.

,,-- R. Coleman, K. Lambeck, and J. X. Mitrovica, 2004: Estimates of the regional distribution of sea level rise over the 1950-2000 period. J. Climate, 17, 2609-2625, doi:10.1175/ 1520-0442(2004)017<2609:EOTRDO>2.0.CO;2.

Clark, J. A., and C. S. Lingle, 1977: Future sea level changes due to West Antarctic ice sheet fluctuations. Nature, 269, 206-209, doi:10.1038/269206a0.

— ture retreat of ice sheets: An effect of $\mathrm{CO}_{2}$ warming of the climate. Sea-Level Changes, M. J. Tooley and I. Shennan, Eds., Institute of British Geographers, 356-370.

Conrad, C. P., and B. H. Hager, 1997: Spatial variations in the rate of sea level rise caused by the present-day melting of glaciers and ice sheets. Geophys. Res. Lett., 24, 1503-1506, doi:10.1029/97GL01338.

Dahlen, F. A., 1976: The passive influence of the oceans upon the rotation of the Earth. Geophys. J. Int., 46, 363-406, doi:10.1111/j.1365-246X.1976.tb04163.x.

Douglas, B., 1997: Global sea rise: A redetermination. Surv. Geophys., 18, 279-292, doi:10.1023/A:1006544227856.

Farrell, W. E., and J. A. Clark, 1976: On postglacial sea level. Geophys. J. Roy. Astron. Soc., 46, 647-667, doi:10.1111/ j.1365-246X.1976.tb01252.x.

Flato, G., and Coauthors, 2013: Evaluation of climate models. Climate Change 2013: The Physical Science Basis, T. F. Stocker et al., Eds., Cambridge University Press, 741-866.

Gelb, A., J. F. Kasper, R. A. Nash, C. F. Price, and A. Sutherland, 1974: Applied Optimal Estimation. MIT Press, 384 pp.

Hamlington, B. D., and P. R. Thompson, 2015: Considerations for estimating the 20th century trend in global mean sea level. Geophys. Res. Lett., 42, 4102-4109, doi:10.1002/2015GL064177.

Hay, C., E. Morrow, R. E. Kopp, and J. X. Mitrovica, 2013: Estimating the sources of global sea level rise with data assimilation techniques. Proc. Natl. Acad. Sci. USA, 110, 3692-3699, doi:10.1073/pnas.1117683109.

,-- and,- 2015 : Probabilistic reanalysis of 20th century sea-level rise. Nature, 517, 481-484, doi:10.1038/nature14093.

Holgate, S. J., 2007: On the decadal rates of sea level change during the twentieth century. Geophys. Res. Lett., 34, L01602, doi:10.1029/2006GL028492.

- and Coauthors, 2013: New data systems and products at the permanent service for mean sea level. J. Coast. Res., 29, 493504, doi:10.2112/JCOASTRES-D-12-00175.1.

Jevrejeva, S., J. C. Moore, A. Grinsted, and P. L. Woodworth, 2008: Recent global sea level acceleration started 200 years ago? Geophys. Res. Lett., 35, L08715, doi:10.1029/2008GL033611.

Kalman, R. E., 1960: A new approach to linear filtering and prediction problems. J. Basic Eng., 82, 35-45, doi:10.1115/1.3662552.

Kopp, R. E., J. X. Mitrovica, S. M. Griffies, J. Yin, C. C. Hay, and R. J. Stouffer, 2010: The impact of Greenland melt on local sea levels: A partially coupled analysis of dynamic and static equilibrium effects in idealized water-hosing experiments. Climatic Change, 103, 619-625, doi:10.1007/s10584-010-9935-1.

_ C. Hay, C. M. Little, and J. X. Mitrovica, 2015: Geographic variability of sea-level change. Curr. Climate Change Rep., $\mathbf{1}$, 192-204, doi:10.1007/s40641-015-0015-5.

Leclercq, P. W., J. Oerlemans, H. J. Basagic, I. Bushueva, A. J. Cook, and R. Le Bris, 2014: A data set of worldwide glacier length fluctuations. Cryosphere, 8, 659-672, doi:10.5194/tc-8-659-2014.

Milne, G. A., W. R. Gehrels, C. W. Hughes, and M. E. Tamisiea, 2009: Identifying the causes of sea-level change. Nat. Geosci., 2, 471-478, doi:10.1038/ngeo544.

Mitrovica, J. X., M. Tamisiea, J. L. Davis, and G. A. Milne, 2001: Recent mass balance of polar ice sheets inferred from patterns of global sea-level change. Nature, 409, 1026-1029, doi:10.1038/ 35059054.

Peltier, W. R., 2004: Global glacial isostasy and the surface of the ice-age Earth: The ICE-5G (VM2) model and GRACE. Annu. Rev. Earth Planet. Sci., 32, 111-149, doi:10.1146/ annurev.earth.32.082503.144359.

Permanent Service for Mean Sea Level, 2014: Tide gauge data. Permanent Service for Mean Sea Level, accessed 31 March 2014. [Available online at http://www.psmsl.org/data/obtaining/.]

Plag, H.-P., 2006: Recent relative sea-level trends: An attempt to quantify the forcing factors. Philos. Trans. Roy. Soc. London, 364A, 821-844, doi:10.1098/rsta.2006.1739.

_ _ and H.-U. Juttner, 2001: Inversion of global tide gauge data for present-day ice load changes. Proc. Second Int. Symp. on Environmental Research in the Arctic and Fifth Ny-Alesund Scientific Seminar, Tokyo, Japan, National Institute of Polar Research, 301-317.

Ray, R. D., and B. C. Douglas, 2011: Experiments in reconstruction twentieth-century sea levels. Prog. Oceanogr., 91, 496-515, doi:10.1016/j.pocean.2011.07.021.

Rhein, M. S. R., and Coauthors, 2013: Observations: Ocean. Climate Change 2013: The Physical Science Basis, T. F. Stocker et al., Eds., Cambridge University Press, 255-315.

Tamisiea, M. J., J. X. Mitrovica, G. A. Milne, and J. L. Davis, 2001: Global geoid and sea level changes due to present-day ice mass fluctuations. J. Geophys. Res., 106, 30849-30863, doi:10.1029/2000JB000011.

Velicogna, I., 2009: Increasing rates of ice mass loss from the Greenland and Antarctic ice sheets revealed by GRACE. Geophys. Res. Lett., 36, L19503, doi:10.1029/2009GL040222. 